\title{
Real-Time On-Line Space Research Laboratory Environment Monitoring With Off-Line Trend and Prediction Analysis
}

\author{
Kenol Jules \\ NASA Johnson Space Center, kenol.jules-1@nasa.gov \\ Paul P. Lin \\ Cleveland State University, p.lin@csuohio.edu
}

Follow this and additional works at: https://engagedscholarship.csuohio.edu/enme_facpub

Part of the Computer and Systems Architecture Commons, and the Space Vehicles Commons How does access to this work benefit you? Let us know!

\section{Publisher's Statement}

NOTICE: this is the author's version of a work that was accepted for publication in Acta Astronautica. Changes resulting from the publishing process, such as peer review, editing, corrections, structural formatting, and other quality control mechanisms may not be reflected in this document. Changes may have been made to this work since it was submitted for publication. A definitive version was subsequently published in Acta Astronautica, 61, 1-6, (06-01-2007); 10.1016/j.actaastro.2007.01.028

\section{Original Citation}

Jules, K., , \& Lin, P. P. (2007). Real-time on-line space research laboratory environment monitoring with offline trend and prediction analysis. Acta Astronautica, 61(1-6), 27-36. doi:10.1016/j.actaastro.2007.01.028

This Article is brought to you for free and open access by the Mechanical Engineering Department at EngagedScholarship@CSU. It has been accepted for inclusion in Mechanical Engineering Faculty Publications by an authorized administrator of EngagedScholarship@CSU. For more information, please contact library.es@csuohio.edu. 


\title{
Real-time on-line space research laboratory environment monitoring with off-line trend and prediction analysis
}

\author{
Kenol Jules ${ }^{\mathrm{a}, *}$, Paul P. Lin ${ }^{\mathrm{b}}$ \\ ${ }^{a}$ NASA-Johnson Space Center, Mail Code OZ-4, 2101 NASA Parkway, Houston, TX 77058, USA \\ ${ }^{\mathrm{b}}$ Cleveland State University, 2121 Euclid Avenue, Cleveland, OH 44115, USA
}

\section{Introduction}

When the Space Shuttle was used as a low gravity platform to perform space research science, acceleration data analysis and interpretation to characterize the reduced gravity environment were performed by one or two data analysts. Of course, the Space Shuttle low gravity missions were of very short duration (a matter of two weeks). Now, with the International Space Station (ISS) in continuous operation, a vast amount

\footnotetext{
* Corresponding author. Tel.: +12812445516 ; fax: +12812448292 .

E-mail address: kenol.jules-1@nasa.gov (K. Jules).
}

of acceleration data is collected daily. This presents a unique challenge to a data analyst. To tackle that problem, an artificial intelligence monitoring system was designed to process, analyze and report the results of the analysis in near real time on a continuous basis. The main objective was to develop an intelligent monitoring system, which not only can classify incoming signals into known patterns, but also identify the unknown ones, in near real time. The system was named the Microgravity Environment Monitoring System (MEMS). As shown in Fig. 1, it was designed to perform the following four tasks:

1. Detect the current vibrating sources onboard the ISS in near real time (Source Detection). 


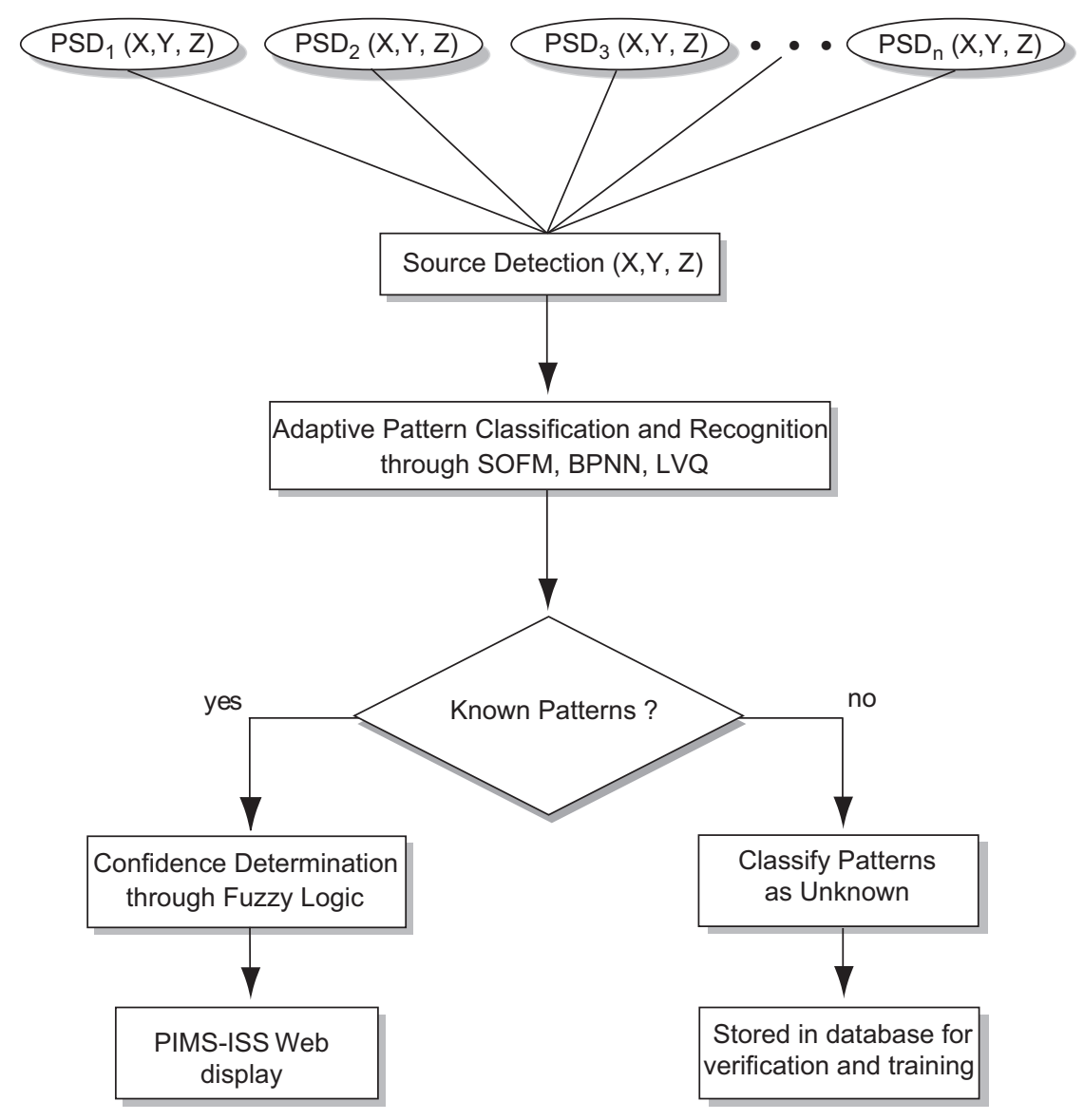

Fig. 1. Microgravity Environment Monitoring System (MEMS) overall architecture.

2. Classify known patterns (Pattern Classification).

3. Recognize unknown patterns (Pattern Recognition).

4. Assess the level of confidence associated with each vibrating source activation (Confidence Determination).

The MEMS extracts, analyzes, and interprets the most salient features of the low gravity environment onboard the ISS as data are downlinked from the ISS for processing. It has three distinct features:

1. On-line monitoring: Performs the task of identifying and assessing the impact of pre-defined parameters associated with a specified system either in situ or remotely in real time.

2. Off-line monitoring: Identifies any unknown parameters or responses associated with a not well defined or not well understood system either in situ or remotely in real time.

3. Trend and prediction analysis: Performs sensitivity analysis and data mining for both pre-defined parameters as well as newly defined ones associated with a specified system.

It is important to note that MEMS main focus is the vibratory regime, but some of the transient activities can be detected as well, depending on the disturbances time duration.

\section{Problem identification and proposed solution}

At first, the problem to be solved sounded simple enough: "identify in near real time, on a continuous basis, all active vibratory disturbance sources, at any time, onboard the ISS from the time domain acceleration data, which are being down-linked from the station". However, once the myriad of other factors were accounted for, complexity arose and soon the problem became a very complicated one to deal with. Many factors were taken into account such as multiple sensors, multiple science locations and vibratory disturbance sources throughout the ISS. In addition, each sensor can be 


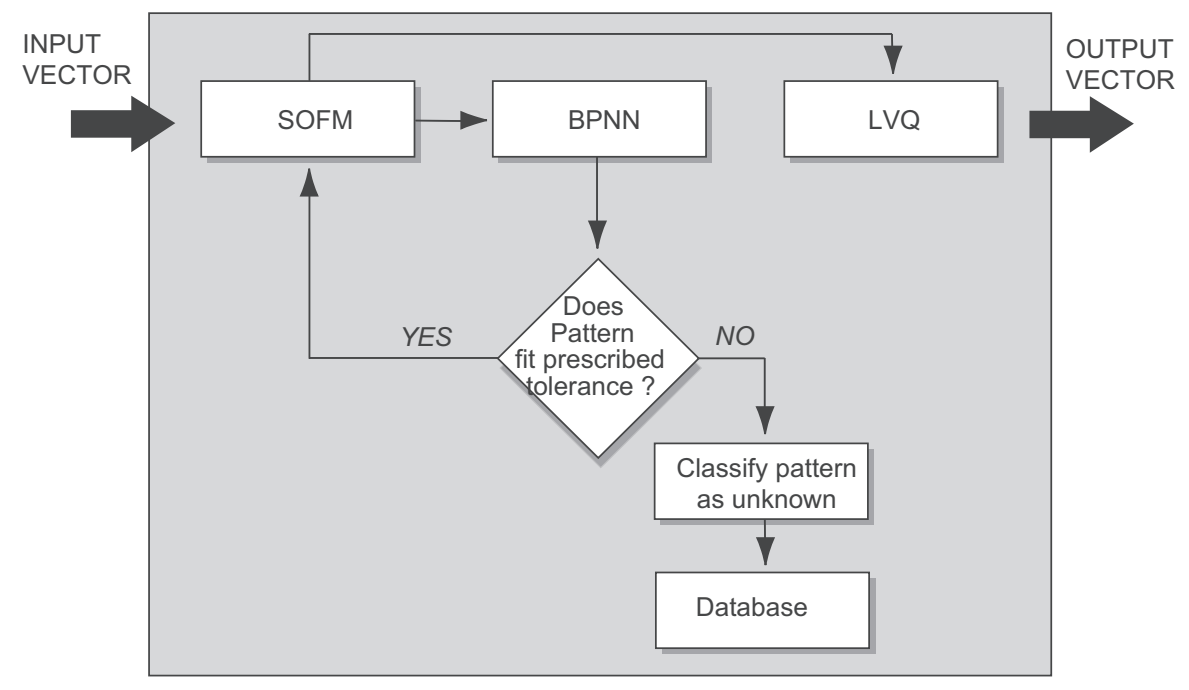

Fig. 2. APCR and fuzzy logic hybrid architecture.

programmed to operate at different cutoff frequencies, which means that some disturbance sources will be measured by more than one sensor (depending on cutoff frequency). Due to the localized nature of the vibratory component, sensors locations must be matched with science locations. Finally, not only must the system classify all the known vibratory sources, in near real time, but also it must recognize the unknown sources as well since the ISS is a new platform and is being built in increments. As such, some disturbance's operating frequencies might change from increment to increment and new ones will surface as new components are added to ISS. Thus, when all of the factors mentioned were taken into account, the problem to be solved became a complex and difficult one. As a consequence, several artificial intelligence techniques were combined, each one to tackle a specific aspect of the problem. The soft computing techniques used, consist of an adaptive pattern classification (see Fig. 2) which is a hybrid of Kohonen's Self-Organizing Feature Map (SOFM) [1], learning vector quantization (LVQ) [2,3], back propagation neural networks (BPNN) [4,5], and fuzzy logic [6,7]. Fuzzy logic and neural networks, the two major soft computing techniques, have very contrasting application requirements. Fuzzy systems are appropriate if sufficient expert knowledge about the process is available, while neural systems are useful if sufficient data are available or measurable. Furthermore, neural networks possess the ability to learn the input-output relationship. A trained neural network provides instant inputto-output mapping with reasonably good accuracy, but without knowledge representation. Fuzzy logic, on the other hand, possesses the ability for knowledge representation and inference, but has no capabilities for automated learning. Thus, fuzzy logic and neural networks compensate each other in terms of information processing.

\section{Soft computing techniques description}

Due to the complexity associated with designing this system, three artificial neural network techniques $[8,9]$ were combined (see Fig. 2) along with fuzzy logic. The principal goal of the SOFM algorithm, an unsupervised learning technique developed by Kohonen, is to transform an incoming signal pattern of arbitrary dimension into a one- or two-dimensional discrete map. It is an unsupervised learning technique. The LVQ is a supervised learning technique that uses higher order information in order to improve the quality of the classifier decision regions. The LVQ algorithm is a stochastic approximation algorithm. This algorithm is designed to minimize the possibility of misclassification since it learns to classify input vectors into the target specified by the user. Since these two techniques are used only to classify known patterns, a BPNN is used to identify patterns, which are not yet known to the system. Fuzzy logic is used to handle multiple sensors reading for the same disturbance and matched the reading with the science and vibrating disturbance source locations (when known) to determine which reading is more relevant to a specific location, using an index known as "degree of confidence", which varies from 0 to 1 . 


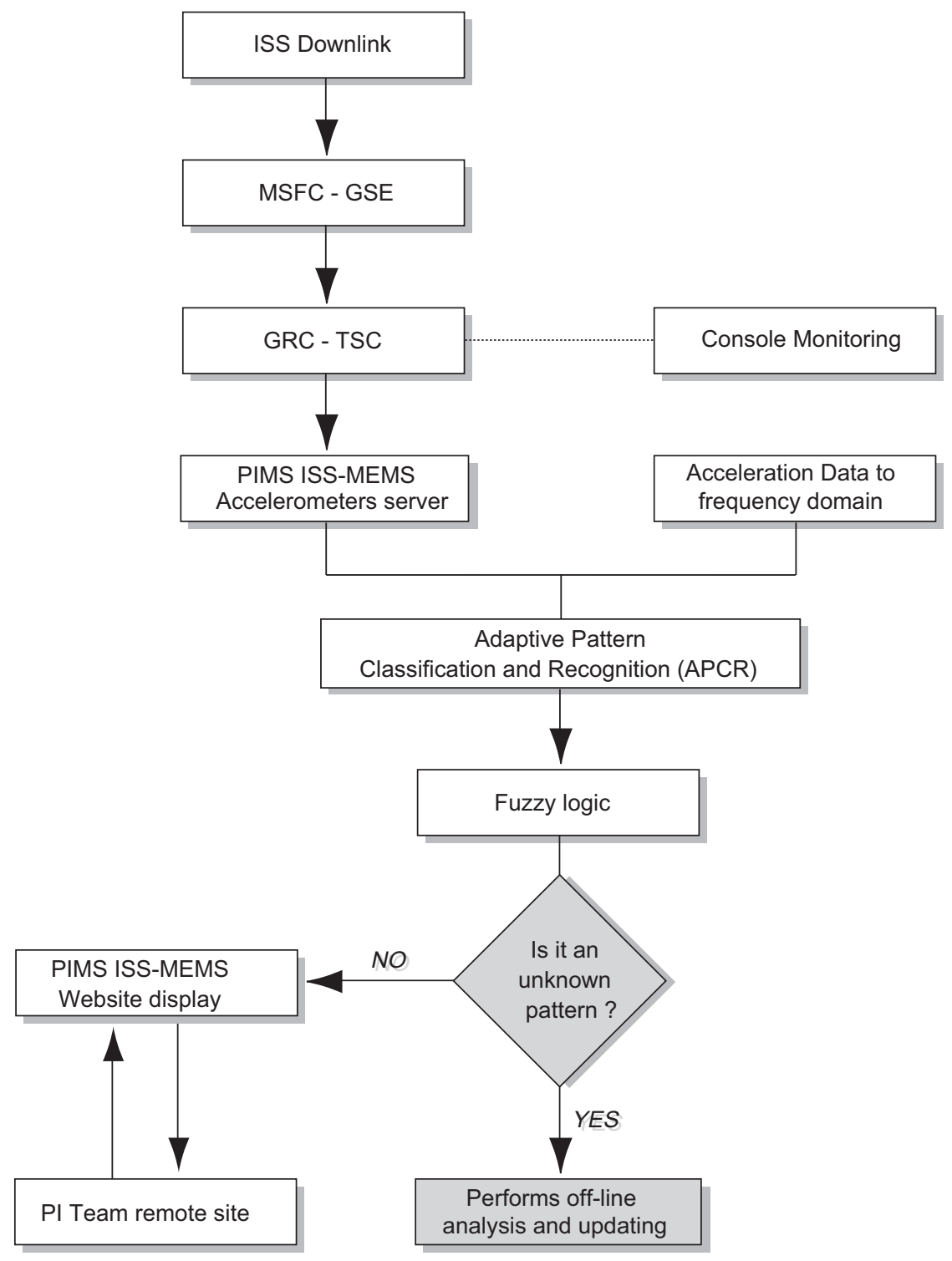

Fig. 3. ISS MEMS on-line processing architecture.

\subsection{Rationale for combining three techniques}

SOFM can group input vectors into several clusters, but cannot group clusters into class, nor can it group different clusters into the same desired target class like LVQ can. This feature (different clusters into the same target class) is very important for both the ISS structural modes and crew exercise events. The structural modes consist of several frequency components spread over a frequency band. The crew exercise has a similar signature with two distinct frequency domain components (body rocking frequency and leg pedaling or footfall frequency). In addition, some vibrating sources exhibit harmonics of their fundamental frequencies. In this case, all the harmonics or the frequency band of a specific event can be lumped into that disturber target class. SOFM and LVQ cannot be used to identify new patterns (unlearned patterns), therefore, BPNN is used to detect the new patterns and store them in a database. Finally, when multiple sensors detect the same event, fuzzy logic is used to determine which sensor reading is more relevant to a specific experiment.

In summary, BPNN is inserted in between SOFM and LVQ to recognize the unknown patterns, while SOFM 
and LVQ are used to classify the known patterns [10], as shown in Fig. 2. In addition, BPNN is used to keep SOFM in check in order to prevent it from misclassifying patterns since one of the shortcomings of SOFM is the potential for misclassifying patterns that fall on the boundary between two clusters due to the fact that SOFM relies solely on Euclidean distance to assign patterns to respective clusters. Fuzzy logic provides data fusion capability and confidence level. The monitoring system is fully automated from data processing/analysis to final decision as to what vibrating sources are active, including degree of confidence associated with the reported results.

\section{System on-line processing}

The on-line system [11] begins with the acquisition of acceleration data using the sensors [12] onboard the station, Fig. 3. The acceleration data received from the sensors are in time domain. They are, then, transformed to frequency domain by means of fast Fourier transform (FFT), from which the so-called power spectral density (PSD) is calculated. The PSD $[13,14]$ is a frequency domain function, which is often used to indicate the dominant frequency components present in the data. PSD analysis is performed on time series data to identify the relative magnitudes of sinusoidal signals that compose the series. The basis of this computation is the Fourier transform [15], which gives an estimate of the distribution of power with respect to frequency in the acceleration signal, and it is used to identify and quantify the vibratory components of the acceleration environment.

The major peak values, Fig. 4, of PSD in the frequency domain represent the operating frequencies of the different vibrating sources, which are to be detected by the monitoring system in order to identify the vibrating sources. MEMS uses the PSD data to detect the characteristic frequency of each disturbance (or pattern) using a peak detector algorithm in order to classify all incoming learned patterns. All unknown patterns are handed over to the off-line mode of MEMS for further analysis.

For each detected relevant peak, MEMS uses the modified Parseval theorem to estimate the RMS acceleration, from which the acceleration magnitude level from the time domain is calculated, for each detected frequency. For each pair of acquired parameter detected (frequency and acceleration), MEMS uses SOFM to assign the pair to its proper cluster after further verification performed by BPNN. If no match is found (not a known pattern), the cluster is transferred to a database reserved for unknown patterns for further analysis

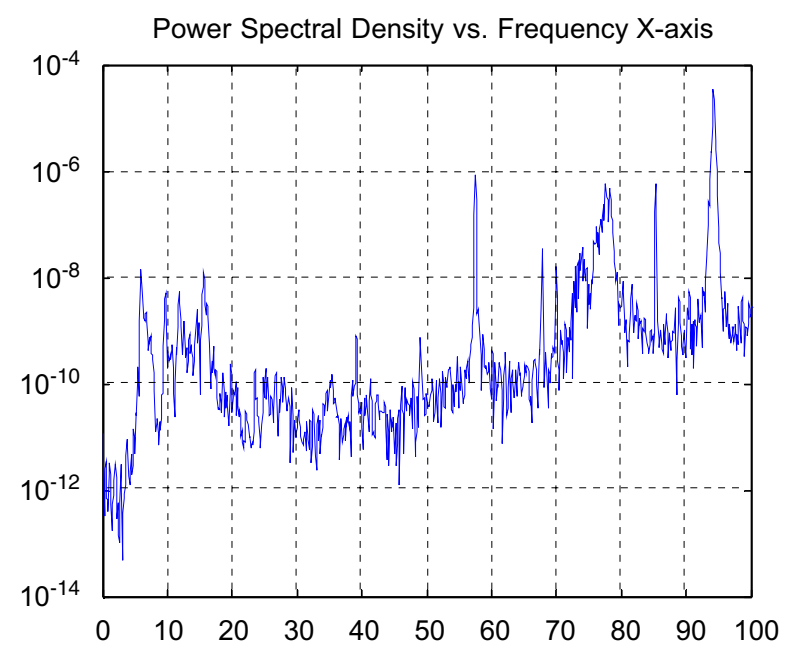

Fig. 4. Power spectral density (PSD) vs. frequency.

(off-line analysis). Once the pair is affirmed, SOFM, sends it to LVQ, which classifies the pair as well as matching the value (frequency and acceleration) of the pair with the name of the pattern (for example, fan or pump) in the known database. Once, the name of the pair is identified, the fuzzy logic process takes over to determine the degree of confidence associated with the detected pattern pair. The result is sent to the web, which can be viewed in near real time using the graphical displays shown in Figs. 5 and 6. Fig. 5 shows a screen capture of the actual MEMS in the on-line mode operation. In Fig. 5 the small rectangle (the first one from the top) is a drop down menu, which lists the different sensor heads available. In this case, sensor number $121 \mathrm{f0} 4$ was selected. Science teams can select the sensor that is most closely located to their science since that sensor will report the most accurate/relevant acceleration magnitude levels for that specific experiment. The second rectangle, moving downward, tells the viewer the current ISS increment. In this case, this screen was captured during ISS increment 9. The next block indicates that the ISS was in non-microgravity mode. Since the ISS is still in the assembly phase, there is no microgravity mode [16] allocation yet. The next block indicates the current day and time in GMT and also the current communication status of ISS with the ground. In this case, it indicates that ISS is in a loss of signal (LOS), which means that ISS is not or cannot downlink data at that moment since there is no satellite coverage. Once there is satellite coverage (i.e., communication with the ground), the LOS will switch to acquisition of signal (AOS), Fig. 6. At that time, the graphical display will start updating every $20 \mathrm{~s}$. The next block of Fig. 5 


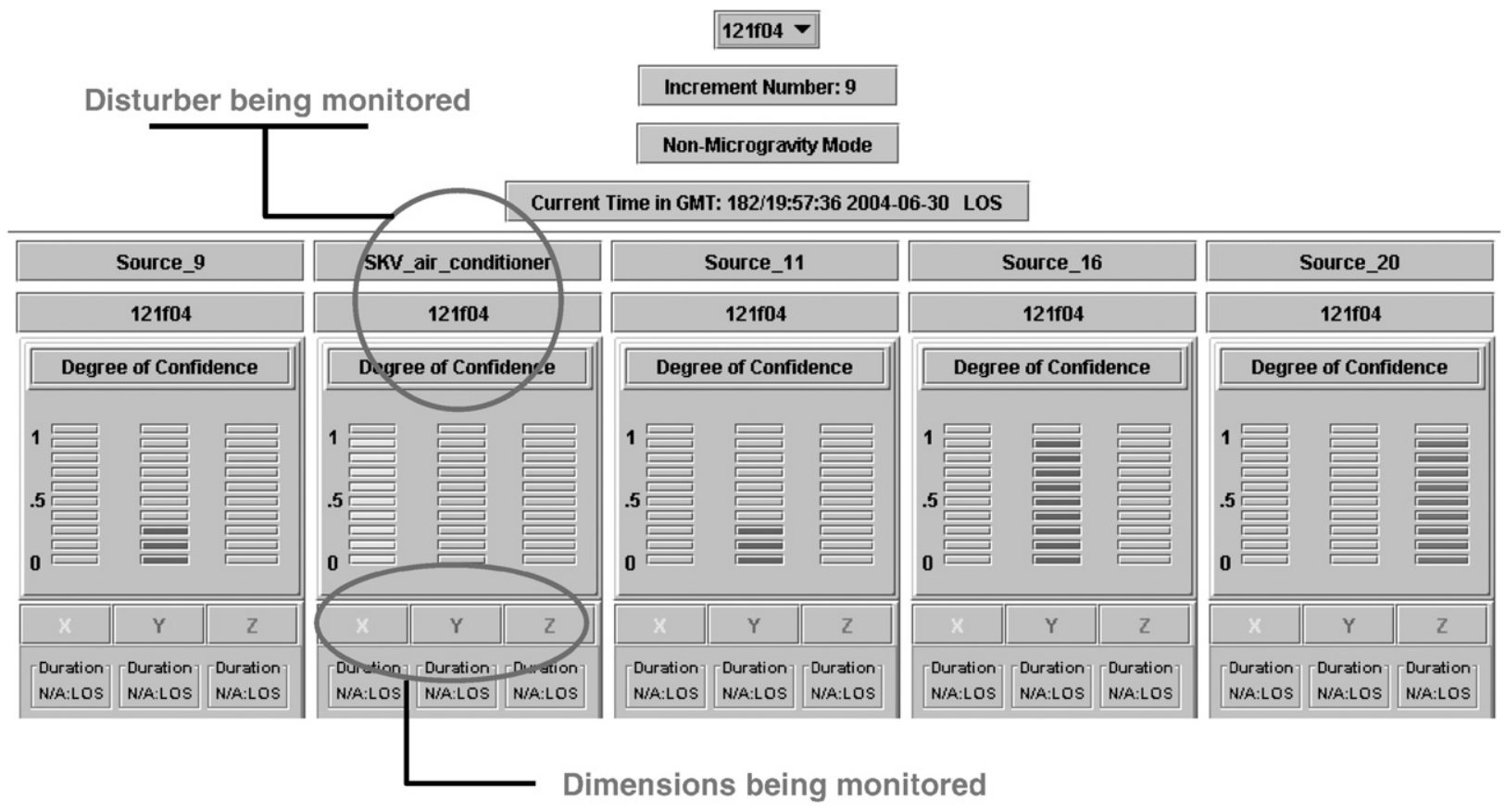

Fig. 5. ISS MEMS real-time displays for increment 9 during an LOS segment.

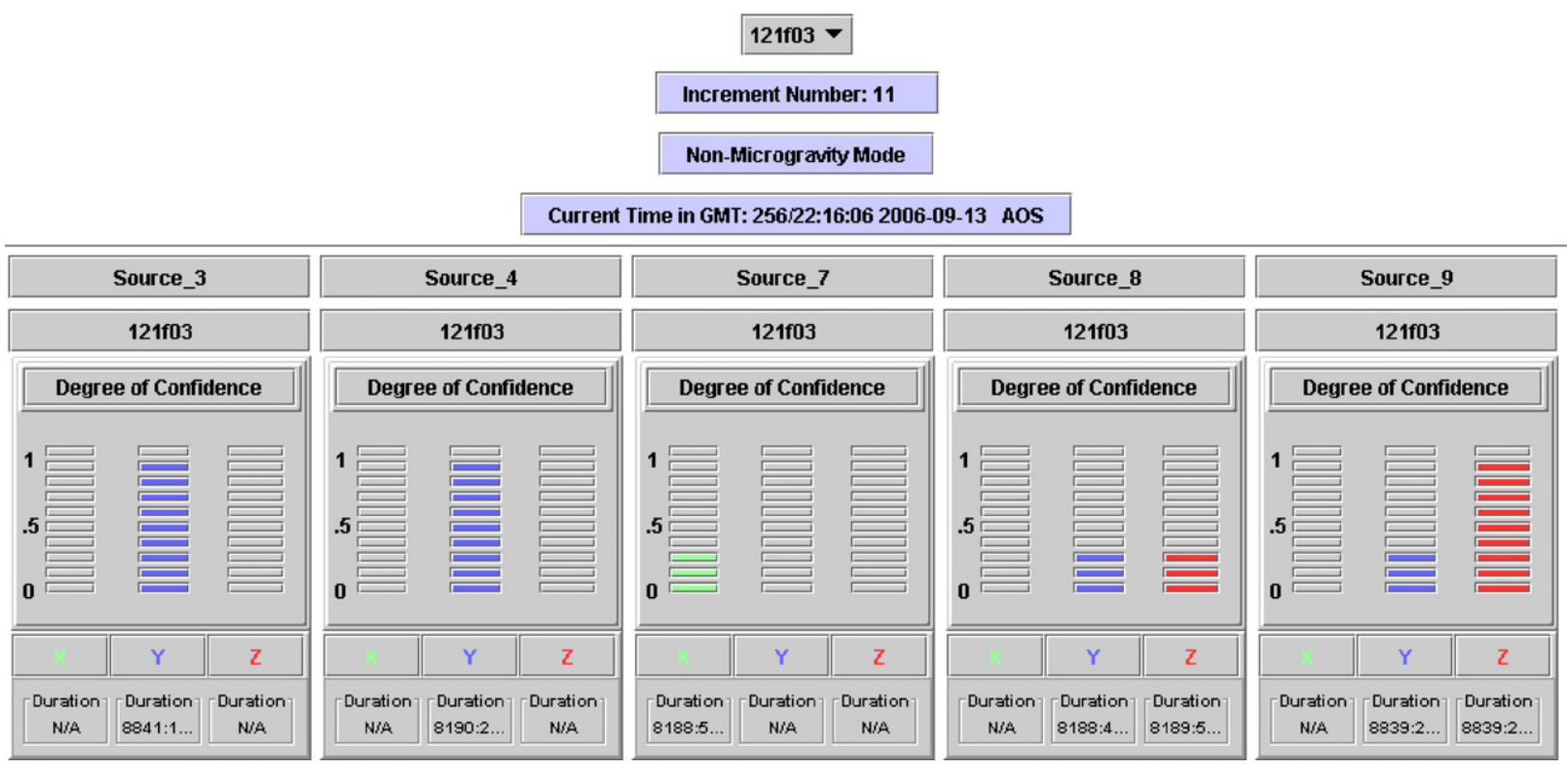

Fig. 6. ISS MEMS real-time displays for increment 11 during an AOS segment.

indicates which vibratory disturbance is being detected. For example, SKV_air_conditioner is a Russian air conditioner. Source_9 or Source_11, for example, indicates that disturbance was detected, but the disturber has not been yet identified-still unknown to the sys- tem. (This identification process will take place as part of the off-line mode. Once this disturber is identified, it will be added to the database, therefore it will become known to the system. Once that is done, next time it is detected again, the correct name will be used instead of 


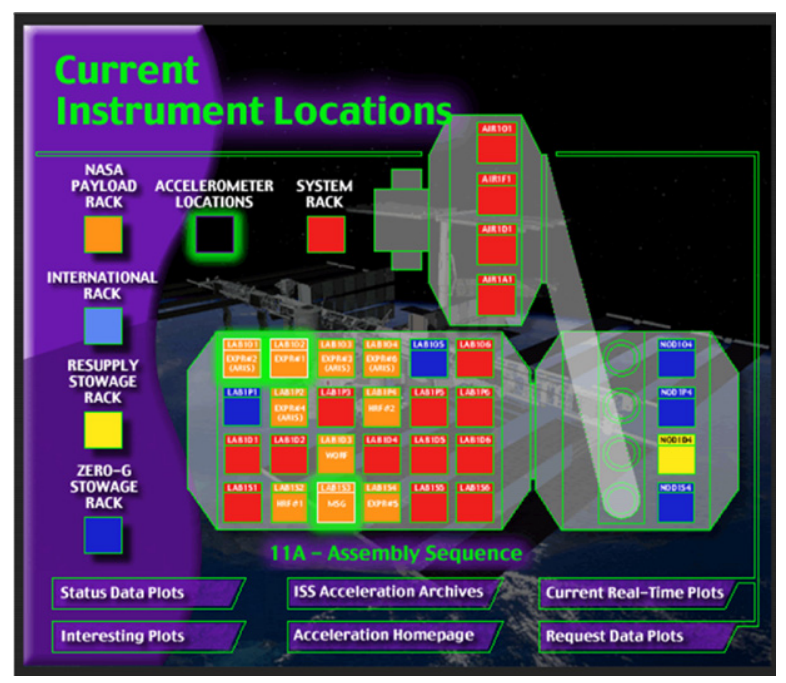

Fig. 7. Pop up window showing location of sensors on ISS.

the word "Source_x", just like SKV_air_conditioner.) The next block indicates the sensor that is reporting this reading. If it is clicked, a window pops up, showing the location of the sensor reporting the current reading, Fig. 7. The next one is the degree of confidence, which goes from 0 to 1 for all three axes of the sensor. A degree of confidence of 0 means that the system is not at all confident that this disturber is active, while a value of 1 means that the system is very confident that the disturber is active and probably located much closer to the sensor reporting that reading than all of the other sensors available. Note that a different level of confidence reading will be shown for each axis due to the nature of the vibratory regime. This is due to the fact that a disturber energy content will most likely be distributed unequally amongst the three axes. If any of the three axes $(X, Y$ or $Z$ ) associated with a specific disturber is clicked, a window pops up showing a log for that disturber. Once a disturber is detected, a $\log$ is automatically created, which keeps useful information such as the name of the disturber, the activation time of the disturber, the average and maximum amplitude, the frequency and how long the disturber has been active. In the event the disturber has just been de-activated, the $\log$ contains the deactivation time or event ending time, Fig. 8. Such a $\log$ is very useful to science teams when analyzing and interpreting their science data since the $\log$ gives them a simplified record of the environment their experiments were exposed to. Knowing the sensitivity of their experiments, the science teams can compare the activity $\log$ of a disturber with any unexplained trend they might come across while analyzing their data

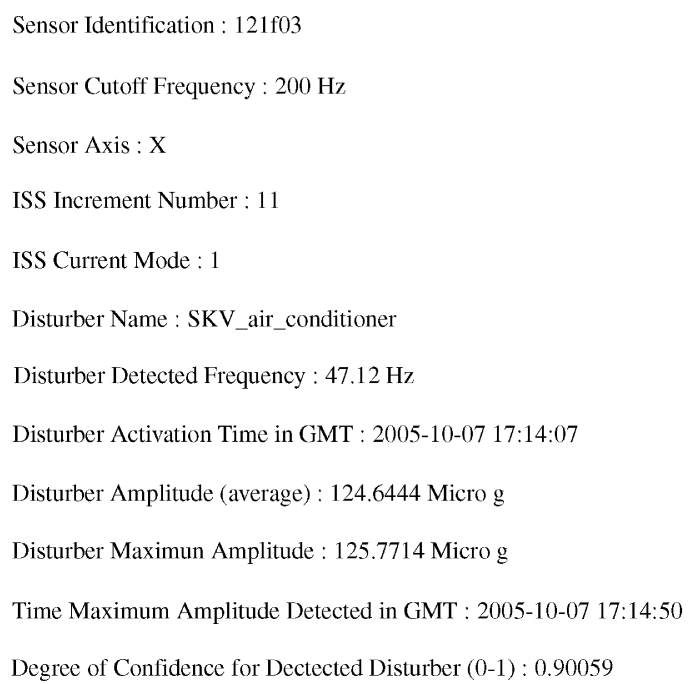

Fig. 8. Example of a log content for an active disturber.

to see if such an event correlates with the time segment they are concerned with. If this log is used appropriately by science teams, it has the potential to accomplish the same function, which had been done in the past by a low gravity environment characterization analyst, albeit it should be used mainly for the vibratory and the transient (dependent of time duration) regimes. The last block (Duration) keeps track of elapsed time when the disturber was detected or activated for all three axes. Fig. 5 shows no data for that entry because the ISS was in LOS, therefore no data were being downlinked due to lack of satellite coverage. Fig. 6, however, shows the time when each disturber was detected by the system as being active on ISS. The elapsed time starts in seconds and then switches to minutes as time increases.

\section{System off-line processing}

The PSD data for each sensor are continuously analyzed. As a result, the detected disturbers are classified as known and unknown patterns. In the off-line mode, Fig. 9, the system identifies and verifies the patterns, which are unknown to the on-line mode (patterns which have not yet learned by the on-line mode). The on-line mode acquires the pattern and then hands it over to the off-line mode for further processing. This relieves the on-line mode from doing any more guesswork, but instead to focus on its main task, which is to identify the learned patterns. Once the hand-over is done, the off-line mode works in the background. It stores certain features of the unknown pattern such as frequency, 


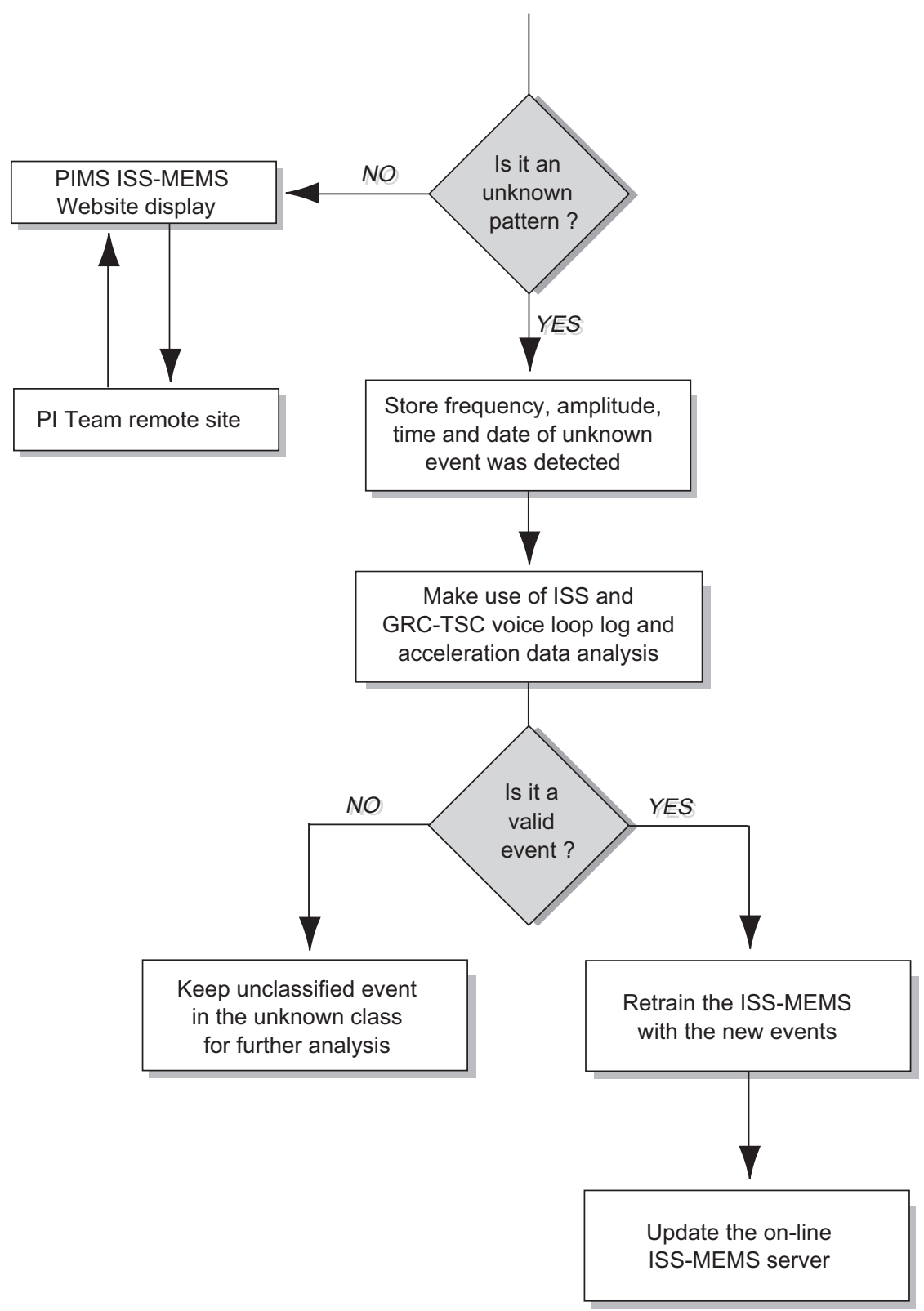

Fig. 9. ISS MEMS off-line processing architecture.

acceleration level, time and date the event was identified to a database.

Generally speaking, a Kohonen's SOFM is designed to perform two tasks: (1) to establish a specified number of clusters and their centers without supervising, and form a SOFM, (2) to classify input patterns according to the established clusters. The first task is accomplished by dividing the given input space into the specified number of clusters based on equal Euclidean distances between any two consecutive cluster centers, and then forms the feature map. However, in doing so, no control can be exercised over the values of cluster centers. In this work, the exact values of cluster centers are the nominal values of frequency and acceleration established by an extensive study from the off-line database. Therefore, there is no need to train a SOFM to establish new cluster centers. In other word, no off-line training is required or needed. In essence, the main MEMS uses the second feature of SOFM in conjunction with LVQ to classify patterns. However, an 
off-line database is required for the continuous learning of the intelligent system. This is the database that was created using the unknown patterns collected by the on-line mode and stored by the off-line mode. After the accumulation of a number of new unknown patterns in the database, the MEMS on-line database is updated so that it can now recognize the newly updated patterns (formerly known as "unknown patterns or disturbers").

\section{Off-line trend analysis and prediction}

The MEMS off-line database can be searched based on an ISS specific increment, such as ISS increment 9-an increment usually lasts about 6 months. It is dictated by crew rotation on ISS [17]; specific disturber, such as Avionic Air Assembly (AAA) fan; specific sensor head, such as $121 \mathrm{f03}$; specific frequency, such as $23.4 \mathrm{~Hz}$; specific sensor axis, such as $X$-axis; specific ISS operating mode, such as microgravity or non-microgravity. Or a search can be made for a combination of all the parameters just mentioned. With such flexibility, science teams can specify a disturber or a frequency that an experiment is sensitive to, then perform a search over the entire period that experiment was operational onboard the ISS. The database will generate a graphic showing how often the specified disturber was active during that increment or increments and the maximum acceleration magnitude levels during the specified time. A frequency-based analysis can be performed as well for a disturber to determine how much that disturber is deviating from its original operating frequency. This can give indication of potential wear and tear of that rotating machinery. Many other usages can be made of this capability in terms of system self-learning or adaptive learning as well as remote system health monitoring and system off-nominal condition reporting. Fig. 10 shows an example of an off-line sensitivity analysis or health monitoring. Three disturbers where chosen at random (SKV_air_conditioner, Source_11 and Source_13) for the $X$-axis of sensor $121 \mathrm{f03}$ for increment 7 . In this case, the frequency option was not chosen, but rather the disturbers were specified. Also, the maximum magnitude levels were chosen (average magnitude levels can be requested as well). Fig. 10 shows the three disturbers maximum magnitude levels during the period for increment 7 when they were active. The $Y$-axis for this graphic represents magnitude level, in $10^{-6} \mathrm{~g}$, while the $X$-axis represents time, in hours. The blue trace is the maximum magnitude for disturber Souce_13 (an unknown disturber at that time) for the six months period of increment 7 . It clearly shows that disturber exhibits an inconsistent signature in terms of magnitude levels during that period. Speculation is that it could be a fan that has multiple operating frequencies and therefore the load varies dependent on the condition within the environment, which in turn affects the magnitude levels. It should be pointed out that a set of data was collected by the same sensor; therefore sensor location has no influence on the magnitude levels. The green trace is for the SKV air conditioner located in the Russian module of ISS. As can be seen, the maximum magnitude levels are consistent (very little variation), except in a few cases. One would expect the response to be so, otherwise that would indicate that there might be some problem with that system since the SKV has a well-defined operating

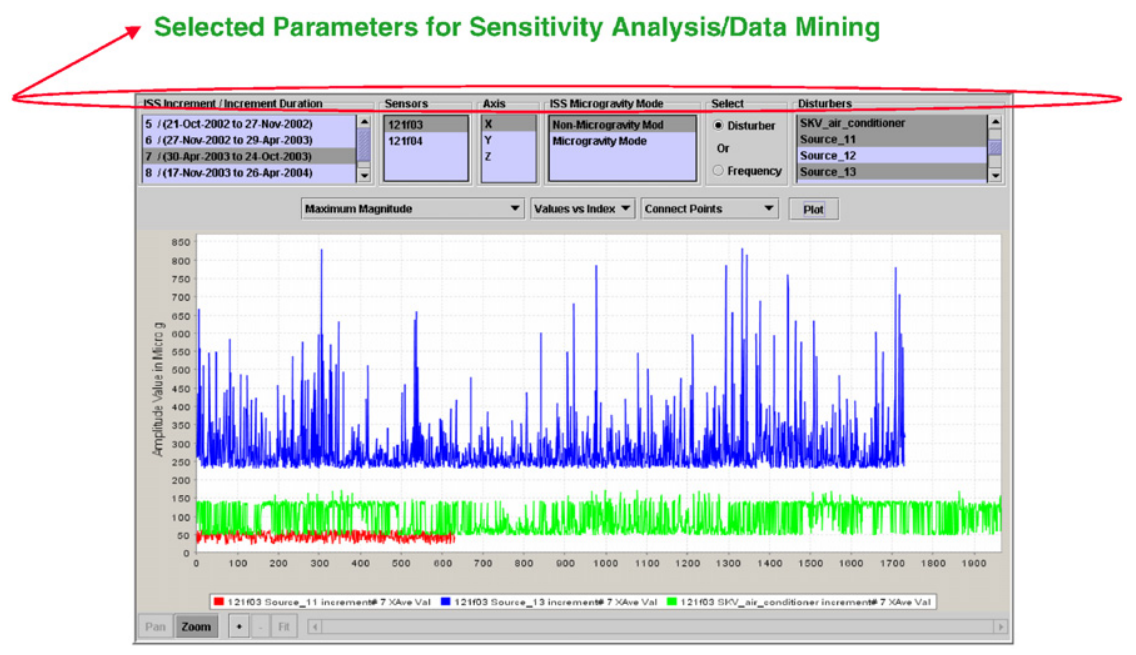

Fig. 10. Example of an off-line sensitive analysis display. 
frequency $(23.4 \mathrm{~Hz})$. Another thing to notice is the fact that the SKV air conditioner was on throughout that increment, which is almost always the case since it is used for crew comfort. The red trace is for another unknown disturber during that increment. Again, the magnitude levels for this unknown disturber were consistent during the time period of its activation. It was activated only one-third of the time during the increment. Speculation is that it could be part of an experiment.

The user can zoom in and out of any selected part on the traces to highlight a specific span of time to see more detail information, it may be for only one day or 30 days at a time. Again, multiple increments can be crossed reference for one or multiple disturbers and for one or multiple sensors as well as multiple sensor axes. This is a very powerful tool for sensitivity analysis and system health monitoring and for knowledge and features extraction from large amount of data. This artificial intelligence monitoring system processed and analyzed about 3 terabytes of acceleration data during the low gravity environment characterization period of ISS for increments $2-11$.

\section{Summary}

The MEMS has demonstrated its capability to automatically detect the vibratory disturbance sources, to correctly identify and classify them. The adaptive pattern recognition and classification approach presented here has the ability to recognize and classify known and unknown patterns, as well as preventing possible patterns misclassification. The two-dimensional pattern (acceleration and frequency) features used gives SOFM an extra dimension, which lessens to some degrees the potential of pattern misclassification. Fuzzy logic is used to exploit the tolerance for imprecision, uncertainty and partial truth, along with the experience of the human experts (by means of fuzzy logic rules), to make intelligent decisions as to what vibrating sources are more relevant to a specific sensor, while neural networks are used to provide very accurate input-output mapping. The artificial intelligence monitoring system discussed in this paper can monitor both in situ and remotely, in real time any change within a defined environment, it may be a biological or a physical system. The monitoring system demonstrates that it can extract specified parameters, measure, monitor, analyze and interpret parameters deviations impact on a somewhat defined system in real time either locally or remotely. Finally, the system can be used for data mining and sensitivity analysis for both pre-defined parameters as well as newly identified ones associated with the system under study or being monitored.

\section{Acknowledgments}

The authors would like to acknowledge the PIMS and SAMS-II project teams for their software and hardware development works, which contributed significantly to the acceleration data used by the MEMS system presented in this paper.

\section{References}

[1] T. Kohonen, Self-organization and Associative Memory, third ed., Springer, New York, Berlin, 1988.

[2] T. Kohonen, Learning vector quantization for pattern recognition, Technical Report TKK-F-A601, Helsinki University of Technology, Finland, 1986.

[3] T. Kohonen, Improved versions of learning vector quantization, in: International Joint Conference on Neural Networks, vol. 1, San Diego, CA, 1990, pp. 545-555.

[4] M.T. Hagan, H.B. Demuth, M. Beale, Neural Network Design, PWS Publishing Company, 1996.

[5] R.P. Lippmann, Pattern classification using neural networks, IEEE Communication Magazine 27 (1989) 47-64.

[6] J.S. Jang, N. Gulley, Application of fuzzy logic in control design, Technical Brief, mathworks Inc., 1996.

[7] C.T. Lin, G.C.S. Lee, Neural Fuzzy Systems, Prentice-Hall, Englewood Cliffs, NJ, 1996.

[8] J.A. Freeman, D.M. Skapura, Neural Network: Algorithms, Applications, and Programming Techniques, Addison-Wesley, Reading, MA, 1991.

[9] S. Haykin, Neural Networks: A Comprehensive Foundation, Prentice-Hall, Englewood Cliffs, NJ, 1994.

[10] P.P. Lin, K. Jules, An intelligent system for monitoring microgravity environment quality on-board the International Space Station, IEEE Transactions on Instrumentation and Measurement 51 (N5) (2002) 1002-1009.

[11] K. Jules, P.P. Lin, Monitoring the microgravity environment quality on-board the International Space Station using soft computing techniques-part I: system design, IAF-00-J.5.06, October 2000, Rio de Janeiro, Brazil.

[12] Space Acceleration Measurement System II, RTS SE Electronic Test Report, SE 121-Q02, SAMS-II 657-RPT1, NASA, December 2000.

[13] J.B.M. Rogers, et al., Analysis techniques for residual acceleration data, NASA-TM 103507, July 1990.

[14] J.B.M. Rogers, K. Hrovat, et al., Accelerometer data analysis and presentation techniques, NASA-TM 113173, September 1997.

[15] E.M. Moskowitz, K. Hrovat, et al., Summary report of mission acceleration measurements for MSL-1, NASA-TM 206979, May 1998.

[16] Microgravity Analysis Integration Team, Microgravity control plan (revision B), Report No. SSP-50036 B, The Boeing Company, Houston, TX, NASA Contract No. NAS15-1000 (DRIVE-16), February 1999.

[17] K. Jules, et al., International Space Station Increment 6/8 Microgravity Environment Summary Report, NASA TM-2006213896, January 2006. 